\title{
Stable CW single-frequency operation of Fabry-Perot laser diodes by self-injection phase locking
}

\author{
Gary L. Duerksen \\ Department of Electrical Engineering, University of Maryland, College Park, MD 20742 \\ $301-405-3678$ \\ gary.duerksen@gsfc.nasa.gov \\ Michael A. Krainak \\ M/C 554, NASA/ Goddard Space Flight Center, Greenbelt, MD 20771 \\ $301-286-2646$ \\ michael.krainak@gsfc.nasa.gov
}

Previously, single-frequency semiconductor laser operation using fiber Bragg gratings has been achieved by two methods: 1) use of the FBG as the output coupler for an anti-reflection-coated semiconductor gain element ${ }^{1}$; 2) pulsed operation of a gain-switched Fabry-Perot laser diode with FBG-optical and RF-electrical feedback. Here, we demonstrate CW single frequency operation from a non-AR coated Fabry-Perot laser diode using only FBG optical feedback.

We coupled a nominal $935 \mathrm{~nm}$-wavelength Fabry-Perot laser diode to an ultra narrow band (18 pm) FBG. When tuned by varying its temperature, the laser wavelength is pulled toward the centerline of the Bragg grating, and the spectrum of the laser output is seen to fall into three discrete stability regimes as measured by the side-mode suppression ratio, shown in figure 1:



Fig. 1: Spectral purity of laser output in frequency stabilized (dashed line), phase stabilized (dotted line), and phaselocked (solid line) regimes as measured by side-mode suppression.

The first regime represents operation frequency-stablilized within one axial mode of the laser cavity. By precisely tuning the laser temperature, we locked an axial mode of the laser to the grating centerline. This second regime constitutes phase-stabilized operation within about a $5 \mathrm{GHz}$ band, as shown in the scanning Fabry-Perot spectrum of figure 2. The third regime is single-frequency operation of the laser, produced when one frequency of laser oscillation is phase-locked to itself.

The phase of the reflected signal from a FBG depends upon the incident phase and its frequency relative to the grating centerline. Using coupled-wave analysis, we demonstrate that in a uniform, single-mode FBG, for incident light whose frequency precisely coincides with the grating centerline frequency, the phase of the reflected signal is conjugate to the incident phase and offset byr $/ 2$. This is analogous to degenerate four-wave mixing. where the grating was written permanently by pump beams during a previous exposure. 
Stable CW single-frequency operation., Gary L. Duerksen \& Michael A. Krainak, page 2

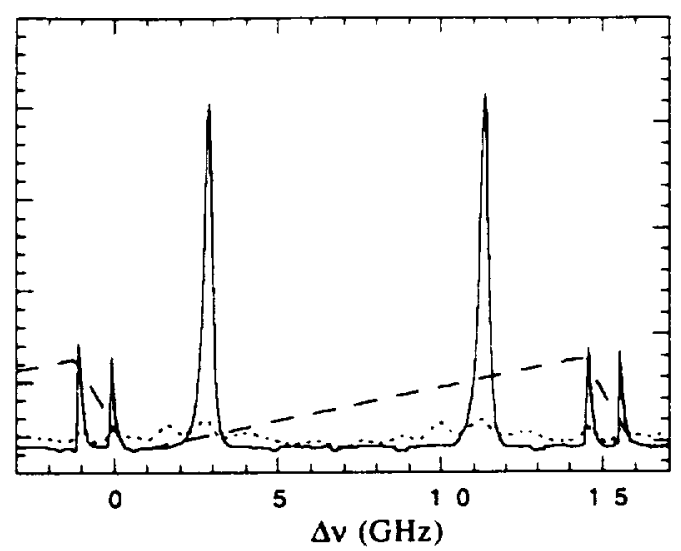

Fig. 2: Scanning Fabry-Perot etalon spectrum of phase-stabilized (dotted line) and phase-locked regimes (solid line) with ramp-voltage trace (dashed line). Phase-locked linewidths are instrument-resolution limited.

Nonlinear gain effects in the laser cause the waveguide mode to propagate with a fixed radius of curvature in the lateral dimension, ${ }^{3}$ engendering a quadratic phase variation at the output. Displacing the feedback signal relative to the output along the lateral axis produces a linear phase deviation between the signals, which we aligned to cancel the 12 phase offset at mode center. This configuration resulted in the phase-locked operation of regime 3 , shown in figure 2 . The linewidth measured by a high-finesse ètalon is less than $25 \mathrm{MHz}$ (instrument limited) at powers above $10 \mathrm{~mW}$.

Mixing between the cavity mode and the injected signal in the nonlinear gain medium creates a linear interferometric pattern in the lateral beam profile that is absent from the transverse profile and only present for phase-locked operation. The fringe spacing, modulation depth and beam displacement show excellent agreement with our model for the mode dynamics in the gain medium. Figure 3 contrasts the profile at the output-facet in both the phase-locked and frequency-stabilized regimes, revealing the spatial displacement of the lateral profile required by phase matching and the interferometric pattern arising from nonlinear mixing.

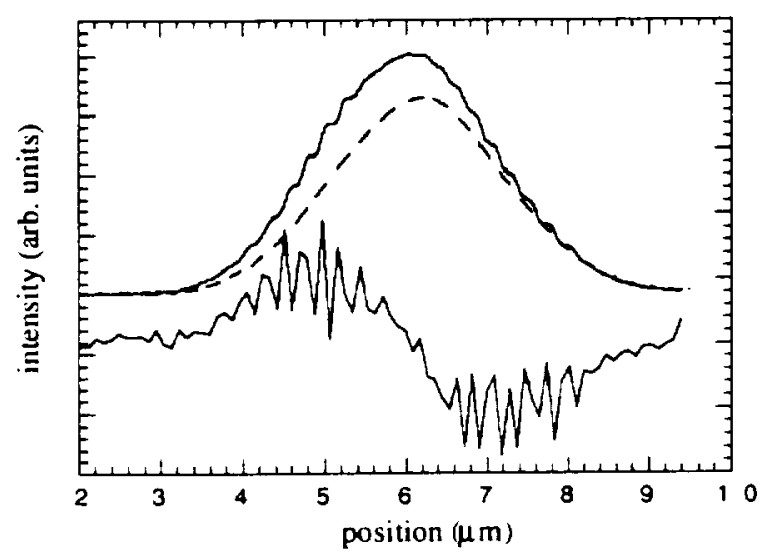

Fig. 3: Single-frequency (solid line) beam profile at the laser facet, and frequency-stabilized (dashed line) beam profiles (from detuning the laser temperature by $10 \mathrm{mK}$ ). The lower line is the slope of the profile.

\section{References}

1. Bird DM. Armitage JR, Kashyap R, et al., Electronics Letters 27, 1115-1116 (1991).

2. Zhao Y, Shu C., IEEE Photonics Technology Letters 9, 1436-1438 (1997).

3. Yariv A, Casperson L, Applied Physics Letters 12. 355-357 (1968). 